\title{
Prevalence of Keratoconus Among a Palestinian Tertiary Student Population
}

\author{
Mohammad M. Shehadeh ${ }^{* 1,3}$, Vasilios F. Diakonis ${ }^{1,2}$, Sara A. Jalil ${ }^{3}$, Rania Younis ${ }^{3}$, \\ Jamal Qadoumi $^{3}$ and Liana Al-Labadi ${ }^{3}$ \\ ${ }^{I}$ Vardinoyannion Eye Institute of Crete (VEIC), Faculty of Medicine, University of Crete, Heraklion, Crete, Greece \\ ${ }^{2}$ Bascom Palmer Eye Institute, Miller School of Medicine, University of Miami, Miami, FL, USA \\ ${ }^{3}$ Faculty of Medicine and health sciences, An-Najah National University, Nablus, Palestine
}

\begin{abstract}
Purpose: To screen for keratoconus and potential associated risk factors in a tertiary student population sample.
Population and Methods: This cross sectional study included 1234 students attending An-Najah National University (Nablus, West Bank, Palestine), that were randomly selected from a total of 20,000 university students. 634 (51.3\%) student participants responded by completing a self-administered questionnaire and were assessed by means of corneal topography. Following initial evaluation, participants were referred for Pentacam evaluation if they demonstrated either a mean keratometry of more than 45 diopters, corneal astigmatism of more than 2 diopters and/or if asymmetric topographic patterns were present. Pentacam images were analyzed by an experienced ophthalmologist based on a number of indices and the participants were classified as normal, keratoconus suspects, and keratoconus patients.

Results: A total of 620 participants (mean age, 20.1 1.6 years) were included in this study, $379(61.1 \%)$ were females and $241(38.9 \%)$ were males. Nine subjects were diagnosed with keratoconus, demonstrating a prevalence of $1.5 \% .52(8.4 \%)$ participants showed at least one abnormal pentacam index, and were considered as $\mathrm{KC}$ suspects.

Conclusion: Keratoconus is a prevalent disease among the tertiary Palestinian student population. This may be related to a combination of genetic and environmental factors. The results of this study signal the need for public health outreach and intervention for keratoconus.
\end{abstract}

Keywords: Keratoconus, pentacam, prevalence, risk factors, topography.

\section{INTRODUCTION}

Keratoconus (KC) is the most common ectatic disorder of the cornea [1], and a major cause of ocular morbidity with significant social and economic impact as the disease affects younger generations [1]. It is an ectatic debilitating corneal disorder characterized by a progressive corneal thinning that results in corneal protrusion, irregular astigmatism, and decreased visual performance $[2,3]$.

The onset of $\mathrm{KC}$ is generally observed during puberty, with a variable amount of progression, which may last until the third or fourth decade of life, when progression usually halts $[4,5]$. This progression is manifested by variable significant loss of visual acuity (VA) which cannot be corrected by spectacles nor contact lenses in advanced cases $[3,6]$. Recent studies found that the onset of $\mathrm{KC}$ is most common in the second and third decades of life. Although it has been reported to develop in later ages [6].

Subclinical or forme fruste $\mathrm{KC}$ is usually an asymptomatic manifestation of $\mathrm{KC}$, which can prove

*Address correspondence to this author at the Ophthalmology Department, An-Najah National University Hospital, Nablus- West Bank- Palestine; Tel: +970 9 2389687; Fax: +970 9 2389684; E-mail: mohammad.sh@najah.edu difficult to diagnose in early stages based on slit-lamp examination alone $[4,7]$. The most sensitive and accurate screening and diagnostic method for subclinical $\mathrm{KC}$ is computer-assisted videokeratography (topography), which allows for the early detection of $\mathrm{KC}$, before clinical findings and patient symptoms become evident $[8,9]$. Early diagnosis of subclinical $\mathrm{KC}$ is essential, as early management may delay or avoid the need for corneal transplantation, and help in preserving functional visual acuity. This cross sectional study will measure the prevalence of $\mathrm{KC}$ among tertiary students, and assess its associated risk factors.

\section{METHODS}

\section{Subject Population}

A quantitative cross sectional study was conducted to determine the prevalence of $\mathrm{KC}$ among all students enrolled in An-Najah National University (ANNU), which at the time of data collection were about 20,000 students. The study was conducted between September 2014 and December 2014. One thousand two hundred and thirty four participants $(\mathrm{N}=1234)$ were recruited to participate in the study using proportionate random sampling technique. A $50 \%$ increase in sample size was utilized to compensate for the expected non-response rate among this population, as reported by a 
similar study conducted in Jerusalem [10]. Students were recruited through notice on the Bulletin boards and web notifications. All registered students included in the study were Palestinian citizens, with no history of corneal pathology other than $\mathrm{KC}$, traumatic corneal scars, nor history of corneal keratoplasty for reasons other than $\mathrm{KC}$.

This research followed the tenets of the Declaration of Helsinki. Ethical approval to conduct this study was obtained from the Institutional Review Board (IRB) committee at ANNU. Informed consent was obtained from all the participants surveyed in the study. All consent forms entailing study objectives and significance were provided to students in Arabic language to ensure they fully understand what their participation requires.

\section{Screening Protocol}

Participants who responded to the research call underwent a self-administered questionnaire. Bilateral topography acquisition and auto-refracto-keratometry was then obtained for all participants, using the color mapping 32 Software for KR-8000PA Supra (8000PA, Topcon, Tokyo, Japan).

Any participant who demonstrated either mean keratometry readings $(\mathrm{K})$ more than 45 diopters (D) [10-12], topographic pattern suspicious of $\mathrm{KC}$ (all irregular patterns except symmetric bowtie (SB), oval, and round patterns) and/or corneal astigmatism of more than 2D [13] was considered to have abnormal topographical indices and was then referred to An-Najah University Hospital for further evaluation by corneal tomography (pentacam). The final analysis and diagnosis of $\mathrm{KC}$ based on tomography results was completed by an experienced ophthalmologist (MSH) and classified according to the criteria shown in Table $\mathbf{1}$ into:
Normal, if all pentacam indices were normal; KC suspect, if pentacam exhibited one or more known abnormal parameters which do not meet the criteria for KC diagnosis [14]; and $\mathrm{KC}$, if a definite diagnosis was confirmed by pentacam.

\section{Data and Statistical Analysis}

The Statistical Package of Social Sciences version 16.0 (SPSS Inc., Chicago, IL, USA) was utilized for data entry and statistical analysis. Only the participants who completed the study were included in data analysis. Chi-square test was used to compare the characteristics of each classification group. A multivariate analysis was used to evaluate the associations with KC. P-values $\leq 0.05$ were considered statistically significant.

\section{RESULTS}

634 participants responded to the research call (response rate $=51.3 \%$ ), of which 14 were excluded as they did not meet the inclusion criteria. The age of the participants ranged between 17 and 27 years (mean $20.1 \pm 1.6)$, and $379(61.2 \%)$ were females and $241(38.8 \%)$ were males. All participants included were of Palestinian origin.

\section{Ocular and Medical Characteristics of the Participants}

One third (30.2\%) of the participants were using spectacles to improve their visual function. The number of participants who were contact lens wearers was $35(5.6 \%)$, of which $33(94.3 \%)$ were using soft contact lenses, while only $2(5.7 \%)$ participants were using rigid gas permeable (RGP) contact lenses.

Table 1. The classification criteria for keratoconus.

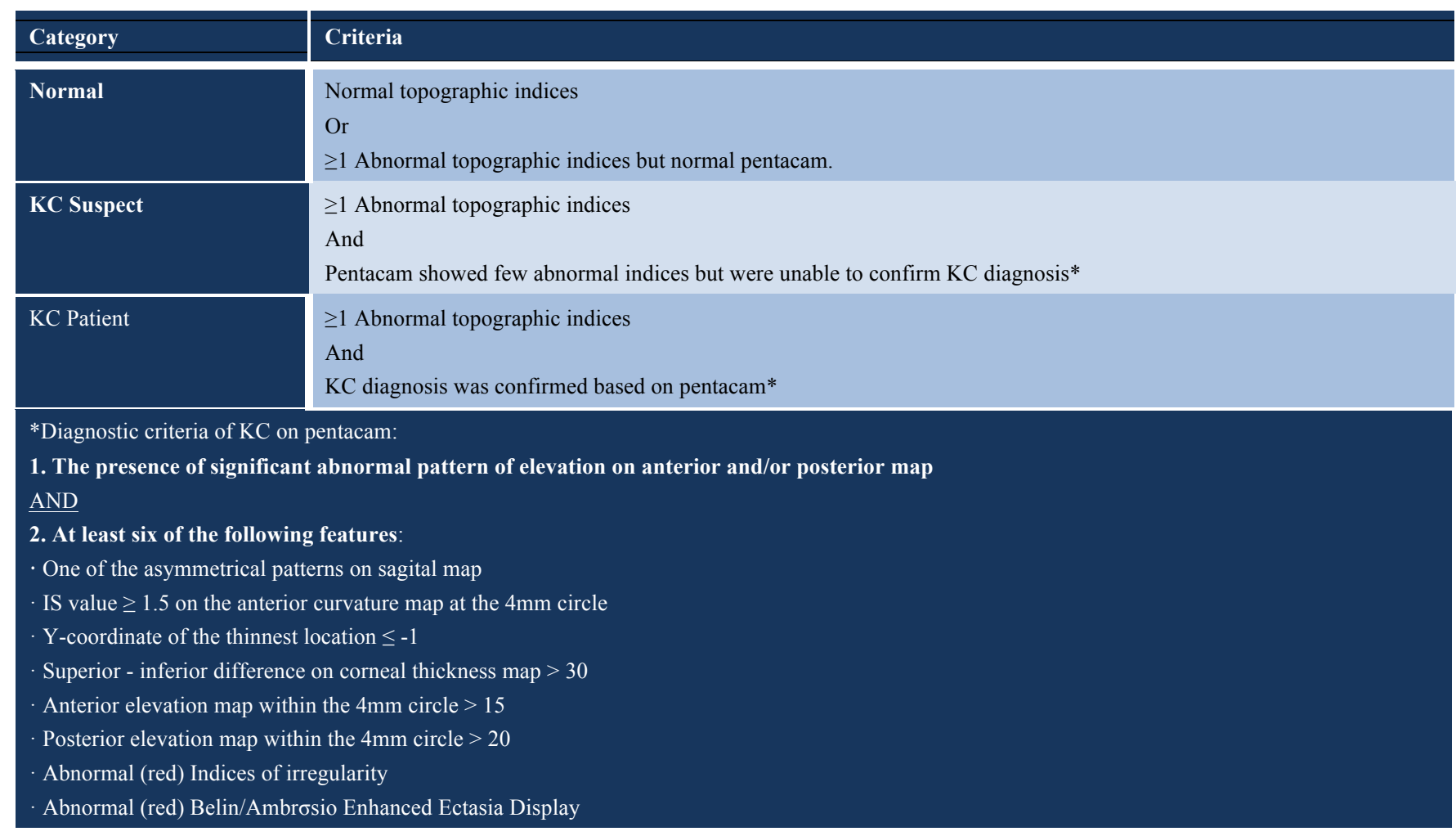


70 (11\%) participants reported daily significant eye rubbing, while about $20 \%$ reported having atopy and 70 $(11 \%)$ participants reported having vernal keratoconjunctivitis (VKC). A positive family history of $\mathrm{KC}$ was reported by $47(7.5 \%)$ participants. Furthermore, 159 (25.6\%) participants had parents who had a consanguineous marriage, of which, $115(72.3 \%)$ parents were first cousins. None of the participants reported having any chronic diseases or genetic conditions associated with KC. A summary of the data concerning the ocular and medical characteristics of participants is shown in Table 2.

Table 2. Ocular and medical characteristics of participants.

\begin{tabular}{|l|l|l|}
\hline Variable & Frequency & Percent (\%) \\
\hline \hline Spectacles use & 191 & $30.3 \%$ \\
\hline Contact lenses & 35 & $5.6 \%$ \\
Soft & 33 & $94.3 \%$ \\
Hard & 2 & $5.7 \%$ \\
\hline Eye surgery & 12 & $1.9 \%$ \\
Refractive corneal & 10 & $84.6 \%$ \\
Cross linking & 2 & $15.4 \%$ \\
\hline Eye rubbing & 72 & $11.6 \%$ \\
\hline History of eye rubbing & 253 & $40.8 \%$ \\
\hline VKC & 65 & $10.5 \%$ \\
\hline Family history of KC & 45 & $7.30 \%$ \\
\hline Consanguinity & 159 & $25.6 \%$ \\
First degree & 115 & $72.3 \%$ \\
Second degree & 24 & $15.1 \%$ \\
Third degree & 20 & $12.6 \%$ \\
\hline Atopy & 126 & $20.3 \%$ \\
\hline Chronic diseases & 0 & $0.00 \%$ \\
\hline
\end{tabular}

\section{Refractive \& Topographic Characteristics}

Participants had a keratometry range between 36.5 to $47.75 \mathrm{D}$, with a mean of $43.24 \pm 1.34 \mathrm{D}$. Thirty-seven subjects (65 eyes) had keratometry values higher than 45 (referral cutoff) in one or both eyes. Corneal astigmatism ranged between 0.00 to $-5.75 \mathrm{D}$ (mean $0.45 \pm 0.44 \mathrm{D}$ ), with 17 subjects (23 eyes) having higher than $2.00 \mathrm{D}$ (referral cutoff) astigmatism. Topographic patterns analyzed showed that the majority $(89 \%)$ of participants had a symmetrical pattern (symmetric bowtie, oval, and round), and the remaining $11 \%$ were divided among the other abnormal patterns.

Based on the referral criteria developed, 91 subjects were referred for further evaluation using pentacam. Of those, 82 (90.11\%) participants responded to the call. Definite KC was found in nine individuals, indicating a prevalence of $1.5 \%$ (Fig. 1). Eight cases presented with bilateral $\mathrm{KC}$, and only one participant had evident keratoconus in only one eye. Two (22\%) subjects were previously diagnosed with $\mathrm{KC}$, while the other seven (78\%) participants were unaware of having $\mathrm{KC}$ before their participation in the study.

Of the 82 participants who underwent additional pentacam analysis, $52(8.4 \%)$ participants were diagnosed as $\mathrm{KC}$ suspects, having an abnormal topographic pattern and at least one abnormal pentacam index. A definite $\mathrm{KC}$ diagnosis could not be established for these participants based on the criteria utilized. Only one suspect was defined as a possible case of post LASIK ectasia.

Participants diagnosed with definite $\mathrm{KC}$ were classified according to the Collaborative Longitudinal Evaluation of Keratoconus (CLEK) study [15], where 3 participants were found to have mild $\mathrm{KC}, 4$ have moderate $\mathrm{KC}$, and 2 have severe $\mathrm{KC}$.

The prevalence of $\mathrm{KC}$ was $1.6 \%$ in females and $1.2 \%$ in males $(p=0.097)$. The presence of family history of KC was found to be significantly associated with a diagnosis of $\mathrm{KC}$ $(p=0.002)$. Participants who had a positive family history were found to have a 13 fold increased risk of having $\mathrm{KC}$, compared to those without family history. Contact lens wear was also associated with 6.8 times higher risk for having $\mathrm{KC}$, but this was not statistically significant $(\mathrm{p}=0.060)$. Although there is an increased risk for $\mathrm{KC}$ with female gender, history of eye rubbing, atopy, and vernal keratoconjunctivitis, none of these associations demonstrated statistical significant difference.

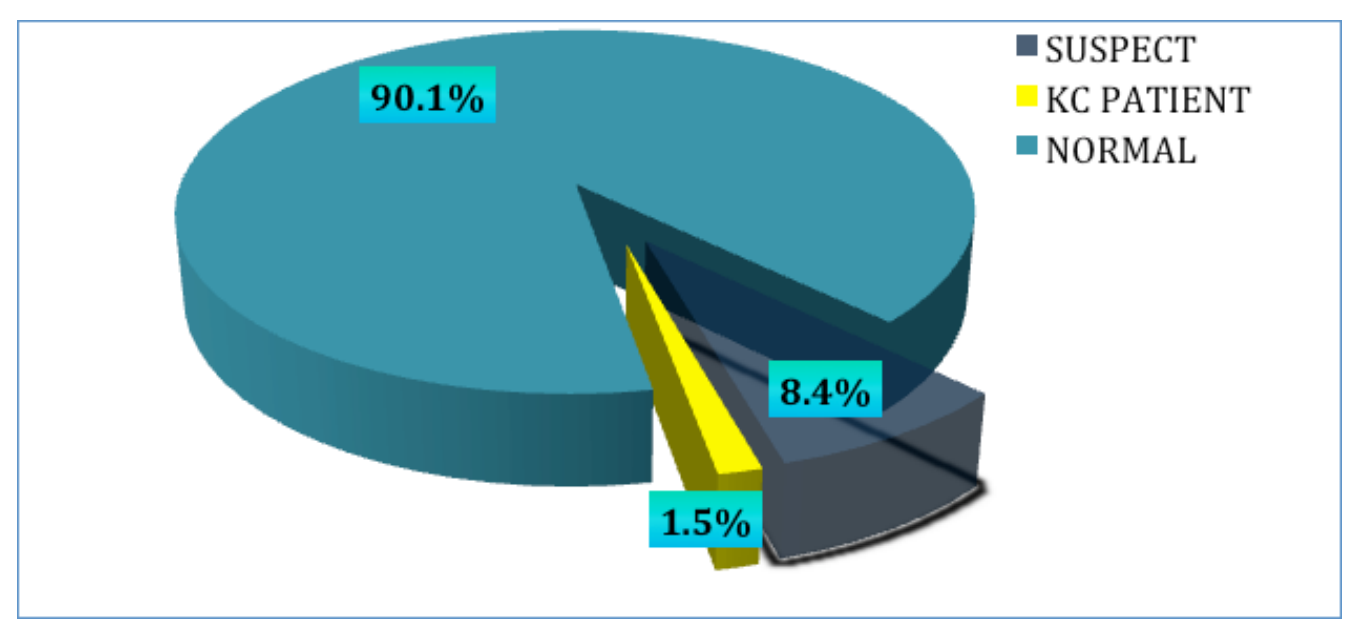

Fig. (1). Prevalence of keratoconus among a tertiary student population. 


\section{DISCUSSION}

In this cross sectional study, the prevalence of $\mathrm{KC}$ was $1.5 \%$ (1500 per 100000$)$. This prevalence is consistent with the results reported in other middle-eastern countries where a much higher prevalence of $\mathrm{KC}$ is demonstrated in comparison to other regions of the world.

The prevalence in our study was lower than the other study conducted in Lebanon (3300/100000) [16]. This may be attributed to the fact that the mean age of participants in the current study was 20.1 years, which was less than the mean age of participants in Lebanon study. Although the mean age in this study is within the period of onset of the disease, a few cases may develop later. This was clear in a study conducted in Tehran, Iran [17], to determine the prevalence of $\mathrm{KC}$ based on topographic maps in a population with ages between 14-81 years. The prevalence of keratoconus was $3.3 \%$. However, the prevalence of $\mathrm{KC}$ was $0.8 \%$ in the $14-29$-year-old age group and $7.5 \%$ in those $\geq 60$ years. The study showed that prevalence of keratoconus significantly increased with age [17]. Another study conducted in Malaysia reported that $26.4 \%$ of KC patients were under 23 years of age, while $52.8 \%$ were in between 23 and 32 years of age [18].

The prevalence of $1.5 \%$ found in this study, represents only definite forms of $\mathrm{KC}$ that matched the diagnostic criteria utilized. Study results showed that $8.4 \%$ of participants with abnormal topography patterns were also found to have many abnormal pentacam indices, but indices values were insufficient to confirm a diagnosis of $\mathrm{KC}$ because they did not meet the diagnostic criteria. Many of these participants may have early form of $\mathrm{KC}$ and a definite diagnosis can only be confirmed with future follow-ups. In the longitudinal study conducted by Li et al. [19], a total of 2501 eyes enrolled at baseline and 1627 eyes were followed for a median of 4.1 years after being classified as normal, $\mathrm{KC}$ suspects, early $\mathrm{KC}$, and $\mathrm{KC}$ based on topography, slit lamp, and retinoscopy examination. Of the originally classified eyes, 277 were considered KC suspects (identified by having asymmetric topographical pattern only), $174 \mathrm{KC}$ suspects continued the follow up and $22(12.6 \%)$ of them developed early KC (abnormal topography and retinoscopy) and $27(15.5 \%)$ developed KC (abnormal topography, retinoscopy, and slit lamp). Thus it is important to follow-up participants to further investigate suspicious $\mathrm{KC}$ tomographic patterns and their risk of developing $\mathrm{KC}$ later in life.

It is important to mention that keratometry of $>45 \mathrm{D}$ is not a sensitive screening protocol for post corneal refractive surgery ectasia, as ectasia may develop with much lower $\mathrm{K}$ values. However, other screening protocols such as abnormal corneal topographic patterns and astigmatism $>2$ D may help to detect the ectasia in its early phase.

It is possible that environmental factors may have contributed to the high prevalence found in the Middle East region where the climate is characterized by dry conditions for most of the year and hot summer periods. On the other hand, in regions such as Denmark, Minnesota, Japan, and the Urals in Russia [20-23] where the weather is much colder with lower average annual temperature, lower $\mathrm{KC}$ prevalence has been reported. Thus it is reasonable to suspect that in the middle-east, sun exposure, which is also associated with higher incidence of atopy and eye rubbing, may play a significant role in KC development [24].

Ethnic differences may also account for the discrepancy in prevalence between the various studies as higher prevalence where mostly reported among Arabs, southAsians (Indian, Pakistani and Bangladeshi) [25, 26], Persians [17], while lower prevalence rates have been reported among the Japanese and white Caucasians. Concerning gender differences, $\mathrm{KC}$ affects both genders, although it is unclear whether significant differences between males and females exist. In our study, there was slightly increased prevalence among females, although this was not statistically significant. However, the predominance of males over females has been noted in recent studies. A study conducted in India [27] reported a difference of 2.6 times higher prevalence in men compared to women. In addition, there was a significant difference between males and females in the Jerusalem study [10] (prevalence 4.91\% vs 1.07\%; P < 0.001 ), which could be due to the significant difference in the mean age between the male group (25.91 years) which was higher than that of the female group (21 years) in their sample.

Family history of $\mathrm{KC}$ has been found to be variable in association with $\mathrm{KC}$ among different studies, ranging between $6 \%$ and $23.5 \%$. In this study, the strongest predictor to be associated with $\mathrm{KC}$ was family history, where $44 \%$ of $\mathrm{KC}$ patients had a positive family history of $\mathrm{KC}$, while only $6.1 \%$ of normal participants reported to have a positive family history of KC. Having a positive family history of KC has around a 13 fold higher risk for developing KC. However, no significant association was found with parental consanguinity.

In the current study and contrary to many previous studies [28], we did not find a significant association between eye rubbing and $\mathrm{KC}$. This difference could be explained on the basis that, in all the studies reporting on eye rubbing, the force of the actual rubbing is not considered, and only the frequency of rubbing is assessed, which is measured on a variety of different scales. In addition, it should be noted that other factors should be taken into account when assessing eye rubbing and its association with $\mathrm{KC}$, such as the method of rubbing (knuckle or finger pads), duration, and seasonal variations.

The association between atopy and keratoconus has been reported extensively. A review of the literature reveals contradictory data in favor of and against this association. Additionally, $\mathrm{KC}$ has been commonly associated with VKC. In this study, $3(33 \%)$ patients reported to have atopic conditions, which was found not to be statistically significant $(\mathrm{p}=0.42) .22 \%$ of $\mathrm{KC}$ patients reported having VKC compared to $10.9 \%$ of the normal participants, although no significant association with VKC was evident. However, most of atopic patients admit that they rubbed their eyes, making it unclear whether atopy itself or the eye rubbing was the most important factor in the etiology of KC.

In this study, there was a significant correlation between contact lens use and KC. It is possible that mechanical trauma induced by eye rubbing and hard contact lens wear, 
acts as an external factor that enhance the progression of the disorder in genetically predisposed individuals.

Our study is limited by its design to enroll participants, a selection bias may have occurred since individuals who knew they had the disease may have refrained from participating in the study because they were under ophthalmic care, while others with visual problems (and no previous diagnosis) may have been more likely to volunteer. This could explain that in this study only $2(22 \%)$ participants with $\mathrm{KC}$ already knew their diagnosis. Nevertheless, the results of the study showed that the prevalence of $\mathrm{KC}$ among tertiary students in Palestine is relatively high, and among the highest in the world. Seventy eight percent of the $\mathrm{KC}$ patients were newly diagnosed in the study, meaning that screening programs may be essential for early $\mathrm{KC}$ detection. Additionally, $8.4 \%$ were $\mathrm{KC}$ suspects, and they could have an early form of $\mathrm{KC}$, and should be followed regularly using tomography. Regulated screening programs for $\mathrm{KC}$ for university students or younger populations, in order to detect $\mathrm{KC}$ in its earliest forms, could prove useful for early diagnosis and management of this corneal entity.

\section{CONFLICT OF INTEREST}

The authors confirm that this article content has no conflict of interest.

\section{ACKNOWLEDGEMENTS}

Declared none.

\section{REFERENCES}

[1] Gatinel D, Saad A. The challenges of the detection of subclinical keratoconus at its earliest stage. Int J Keratoco Ectatic Corneal Dis 2012; 1(1): 36-43.

[2] Ramos-López D, Martínez-Finkelshtein A, Castro-Luna GM, et al. Screening subclinical keratoconus with Placido-based corneal indices. Optom Vis Sci 2013; 90(4): 335-43.

[3] Romero-Jiménez M, Santodomingo-Rubido J, Wolffsohn JS. Keratoconus: a review. Cont Lens Anterior Eye 2010; 8; 33(4): 157-66.

[4] Vazirani J, Basu S. Keratoconus: current perspectives. Clin Ophthalmol 2013; 7: 2019-30.

[5] Wheeler J, Hauser MA, Afshari NA, Allingham RR, Liu Y. The genetics of keratoconus: a review. J Microsc 2012; Suppl 6: 001.

[6] Assiri A, Yousuf B, Quantock A, Murphy P. Incidence and severity of keratoconus in Asir province, Saudi Arabia. Br J Ophthalmol 2005; 89(11): 1403-6.

[7] Carlson AN. Keratoconus: time to rewrite the textbooks. Rev Ophthalmol 2009; 16: 66.

[8] Wygledowska-Promieńska D, Zawojska I. Use of AutoTopograf TMS-3 keratoconus screening program for keratoconus detection. Klinika Oczna 1999; 102(4): 237-40.
[9] Saad A, Gatinel D. Topographic and tomographic properties of forme fruste keratoconus corneas. Invest Ophthalmol Vis Sci 2010; 51(11): 5546-55.

[10] Millodot M, Shneor E, Albou S, Atlani E, Gordon-Shaag A. Prevalence and associated factors of keratoconus in Jerusalem: a cross-sectional study. Ophthalmic Epidemiol 2011; 18(2): 91-7.

[11] Holladay TJ. Keratoconus detection using corneal topography. J Refract Surg 2009; 25(10): 958.

[12] John AK, Asimellis G. Revisiting keratoconus diagnosis and progression classification based on evaluation of corneal asymmetry indices, derived from Scheimpflug imaging in keratoconic and suspect cases. Clin Ophthalmol 2013; 7: 1539.

[13] Serdarogullari H, Tetikoglu M, Karahan H, Altin F, Elcioglu M. Prevalence of keratoconus and subclinical keratoconus in subjects with astigmatism using pentacam derived parameters. J Ophthalmic Vis Res 2013; 8(3): 213.

[14] Belin MW, Kim JT, Zloty P, Ambrosio Jr R. Simplified nomenclature for describing keratoconus. Int $\mathrm{J}$ Keratoco Ectatic Corneal Dis 2012; 1: 31-5.

[15] Zadnik K, Barr JT, Edrington TB, et al. Baseline findings in the Collaborative Longitudinal Evaluation of Keratoconus (CLEK) Study. Invest Ophthalmol Vis 1998; 39(13): 2537-46.

[16] Waked N, Fayad A, Fadlallah A, El Rami H. Dépistage du kératocône dans une population universitaire au Liban. J Fr Ophtalmol 2012; 35(1): 23-9.

[17] Hashemi H, Khabazkhoob M, Fotouhi A. Topographic keratoconus is not rare in an Iranian population: the Tehran eye study. Ophthalmic Epidemiol 2013; 20(6): 385-91.

[18] Mohd-Ali B, Abdu M, Yaw CY, Mohidin N. Clinical characteristics of keratoconus patients in Malaysia: a review from a cornea specialist centre. J Optometry 2012; 5(1): 38-42.

[19] Li X, Yang H, Rabinowitz YS. Keratoconus: classification scheme based on videokeratography and clinical signs. J Cataract Refract Surg 2009; 35(9): 1597-603.

[20] Nielsen K, Hjortdal J, Nohr AE, Ehlers N. Incidence and prevalence of keratoconus in Denmark. Acta Ophthalmol Scand 2007; 85(8): 890-2.

[21] Kennedy RH, Bourne WM, Dyer JA. A 48-year clinical and epidemiologic study of keratoconus. Am J Ophthalmol 1986; 101(3): 267-73.

[22] Tanabe U, Fujiki K, Ogawa A, Ueda S, Kanai A. Prevalence of keratoconus patients in Japan. Nippon Ganka Gakkai Zasshi 1985; 89(3): 407-11.

[23] Gorskova EN, Sevost'ianov EN. Epidemiology of keratoconus in the Urals. Vestn Oftalmol 1998; 114(4): 38-40.

[24] Kenney MC, Brown DJ. The cascade hypothesis of keratoconus. Cont Lens Anterior Eye 2003; 26(3): 139-46.

[25] Georgiou T, Funnell CL, Cassels-Brown A, O'Conor R. Influence of ethnic origin on the incidence of keratoconus and associated atopic disease in Asians and white patients. Eye 2004; 18(4): 37983 .

[26] Pearson AR, Soneji B, Sarvananthan N, Sandford-Smith JH. Does ethnic origin influence the incidence or severity of keratoconus? Eye 2000; 14( Pt 4): 625-8.

[27] Jonas JB, Nangia V, Matin A, Kulkarni M, Bhojwani K. Prevalence and associations of keratoconus in rural maharashtra in central India: the central India eye and medical study. Am J Ophthalmol 2009; 148(5): 760-5.

[28] Weed K, MacEwen C, Giles T, Low J, McGhee C. The Dundee University Scottish Keratoconus study: demographics, corneal signs, associated diseases, and eye rubbing. Eye 2008; 22(4): 53441 . 